\title{
A journey into Bitcoin metadata
}

\author{
Massimo Bartoletti · Bryn Bellomy · Livio Pompianu
}

Received: date / Accepted: date

\begin{abstract}
Besides recording transfers of currency, the Bitcoin blockchain is being used to save metadata - i.e. arbitrary pieces of data which do not affect transfers of bitcoins. This can be done by using different techniques, and for different purposes. For instance, a growing number of protocols embed metadata in the blockchain to certify and transfer the ownership of a variety of assets beyond cryptocurrency. A point of debate in the Bitcoin community is whether metadata negatively impact on the effectiveness of Bitcoin with respect to its primary function. This paper is a systematic analysis of the usage of Bitcoin metadata over the years. We discuss all the known techniques to embed metadata in the Bitcoin blockchain; we then extract metadata, and analyse them from different angles.
\end{abstract}

Keywords Bitcoin, blockchain, measurements

\section{Introduction}

The last few years have witnessed an increasing interest in Bitcoin, the first and most widespread decentralised cryptocurrency $[40,48]$. Bitcoin records currency transactions in a public, append-only data structure - the so-called blockchain. The blockchain is maintained by

\section{Bartoletti}

Università degli Studi di Cagliari, Dipartimento di Matematica e Informatica, Via Ospedale, 72, 09124 Cagliari, Italy

E-mail: bart@unica.it

B. Bellomy

ConsenSys, 49 Bogart St., Brooklyn, NY 11206

E-mail: bryn.bellomy@consensys.net

L. Pompianu

Università degli Studi di Cagliari, Dipartimento di Matematica e Informatica

E-mail: livio.pompianu@unica.it a peer-to-peer network, following a consensus protocol which ensures that tampering with the past transactions is computationally unfeasible [42].

The immutability of the Bitcoin blockchain, together with its openness, have inspired the development of new applications, that - going beyond transfers of currency - certify the existence of documents [16,21, 25], track the ownership of assets $[12,18,19]$, run smart contracts $[14,29,36]$ or perform other useful tasks. These applications exploit Bitcoin transactions to "piggy-back" their metadata, i.e., pieces of data which are not inherent to currency transfers, but are needed to implement their application logic.

A debate about scalability has been taking place in the Bitcoin community over the last few years $[1,22,23]$. In particular, users argue over whether the blockchain should allow for storing these spurious data. Besides quantifying the impact of metadata on the effectiveness of Bitcoin, many other relevant aspects on the usage of metadata are still worth of investigation.

This paper is a systematic survey on the usage of metadata in Bitcoin, based on the analysis of the first 480,000 blocks in the blockchain, i.e. $\sim 245 \mathrm{~K}$ transactions collected until 2017/08/10. Our main contributions can be summarised as follows:

1. We survey the existing techniques for embedding metadata in the Bitcoin blockchain, identifying 11 distinct ones. We compare these techniques, discussing their side effects, and we compare their evolution over time, quantifying the amount of metadata embedded through them.

2. We search the blockchain for metadata, and we parse them to infer the intended usage. To this purpose, we consider both metadata as single units of information, and as aggregates of pieces scattered through the blockchain (e.g., images). Overall, we recognise 7 
different types of metadata. We quantify the amount and size of metadata by type.

3. We identify 45 distinct protocols which are used by applications to embed metadata in the blockchain. We classify them according to their application domain, and we measure the amount of metadata they produced. We analyse the correlation between embedding techniques, metadata types and protocols.

4. We compare the size of the extracted metadata with the overall size of the blockchain, and we investigate peaks of metadata that occurred over the years.

5. We make available a public dataset of metadata extracted from the blockchain [35], as well as the tools we have developed for our analyses [33,38].

Structure of the paper. Section 2 is a minimalistic introduction to Bitcoin, containing all the technical background needed in the subsequent sections. In Section 3 we show and compare the techniques to embed metadata in the blockchain, presenting several statistics on their usage. In Section 4 we illustrate some techniques to parse metadata and reconstruct the original content; then, we categorize and quantify the metadata extracted from the blockchain. In Section 5 we investigate the protocols which embed metadata, we classify them, and quantify the volume of metadata produced by each protocol. Section 6 discusses how the usage of metadata impacts on the Bitcoin blockchain. Finally, Section 7 discusses some related works, and Section 8 draws some conclusions.

\section{Background on Bitcoin}

Bitcoin [48] is a decentralized infrastructure to exchange virtual currency - the bitcoins. Users interact with Bitcoin through addresses, by publishing transactions that transfer bitcoins from one address to another. The log of all transactions is recorded on the blockchain, a public and immutable data structure maintained by the nodes of the Bitcoin network. A subset of nodes, called miners, gather the transactions sent by users, aggregate them in blocks, and try to append these blocks to the blockchain. A consensus protocol based on moderatelyhard "proof-of-work" puzzles is used to resolve conflicts that may happen when different miners concurrently try to extend the blockchain, or when some miner attempts to append a block with invalid transactions. Ideally, the blockchain is globally agreed upon, and free from invalid transactions, unless the adversary controls the majority of the computational power of the network $[31,42,44]$. The security of the consensus protocol relies on the assumption that miners are rational, i.e. that following the protocol is more convenient than trying to attack it. To make this assumption hold, miners receive some economic incentives for performing the time-consuming computations required by the protocol. Part of these incentives is given by the fees paid by users upon each transaction.

\subsection{Transactions}

To illustrate how transfers of bitcoins work, we consider two transactions $T_{0}$ and $T_{1}$, which we represent graphically as follows:

\begin{tabular}{|l|l|}
\hline \multicolumn{1}{|c|}{$T_{0}$} & \multicolumn{1}{c|}{$T_{1}$} \\
\hline $\begin{array}{l}\text { previous transaction: } \cdots \\
\text { in-script: } \cdots\end{array}$ & $\begin{array}{l}\text { previous transaction: } T_{0} \\
\text { in-script: } \operatorname{sig}_{k}(\bullet)\end{array}$ \\
\hline value: $v_{0}$ & value: $v_{1}$ \\
out-script $(T, \sigma): \operatorname{ver}_{k}(T, \sigma)$ & out-script: $\cdots$ \\
\end{tabular}

The transaction $T_{0}$ contains $v_{0}$ Satoshis ( 1 bitcoin $=10^{8}$ Satoshis). A user can redeem this amount by publishing a transaction (e.g., $T_{1}$ ), whose previous transaction field contains the identifier of $T_{0}$ (displayed just as $T_{0}$ in the figure), and whose in-script field makes the out-script ${ }^{1}$ of $T_{0}$ evaluate to true. When this happens, the value of $T_{0}$ is transferred to the new transaction $T_{1}$, and $T_{0}$ becomes unredeemable. A subsequent transaction can then redeem $T_{1}$ likewise. In the transaction $T_{0}$ above, out-script checks that $\sigma$ is a valid signature (made with the key $k$ ) of the redeeming transaction. We denote with $\operatorname{ver}_{k}(T, \sigma)$ the signature verification function, and with $\operatorname{sig}_{k}(\bullet)$ the signature of the enclosing transaction, including all the parts of the transaction but its in-script (obviously, because it contains the signature itself).

Now, assume that $T_{0}$ is redeemable on the blockchain when someone tries to append $T_{1}$. This is possible if $v_{1} \leq v_{0}$, and the out-script of $T_{0}$, applied to $T_{1}$ and to the signature $\operatorname{sig}_{k}(\bullet)$, evaluates true.

The previous example shows the simple case of transactions with only one input and one output. In general, transactions have the form displayed in Figure 1. First, there can be multiple inputs and outputs (denoted with array notation in the figure): in-counter specifies the number of inputs, and out-counter that of outputs. Each input (resp. output) has its own in-script (resp. out-script). The two fields in-script length and out-script length denote the size of in-script and out-script, respectively. Since each output can be redeemed independently, previous transaction fields

1 The fields in-script and out-script are called, respectively, scriptPubKey and scriptSig in the Bitcoin wiki. 


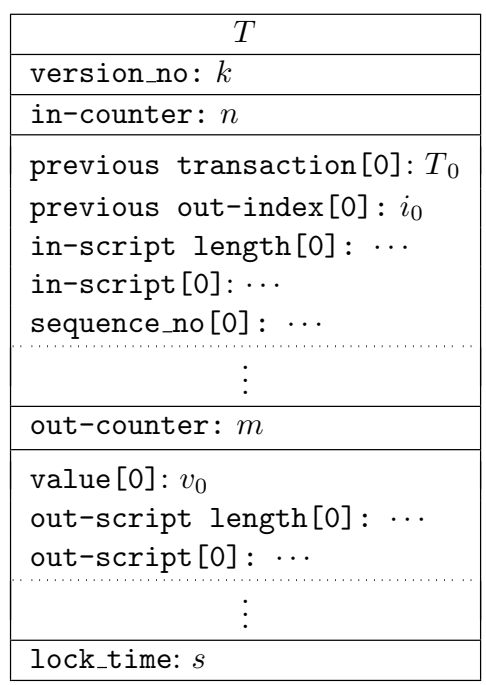

Fig. 1: A Bitcoin transaction.

must specify which one they are redeeming (in the figure, previous out-index). A transaction with multiple inputs redeems all the (outputs of) transactions in its previous transaction fields, by providing a suitable in-script for each of them. The lock_time field specifies the earliest moment in time when the transaction can appear on the blockchain. The version_no field is currently set to 1 . Transaction inputs contain also a 4-bytes field called sequence_no. Normally its value is $0 x F F F F F F F$, and it is ignored unless the transaction lock_time is greater than 0 [11].

In order for $T$ to be appended to the blockchain, a few conditions must be satisfied: for instance, for each $k<n$, the out-script of the $i_{k}$-th output of the transaction $T_{k}$ must evaluate to true when fed with the value in in-script [k]; further, none of the inputs must have been redeemed yet, and the sum of the values of all the redeemed outputs must be greater than or equal to the sum of the values of all outputs in $T$ (see [30] for a formal specification).

The Unspent Transaction Output set (in short, UTXO set) is the set of redeemable outputs of all transactions in the blockchain.

\subsection{Scripts}

Bitcoin scripts $[10,11]$ are programs in a stack-based language featuring a limited set of logic, arithmetic, and cryptographic operators (but without loops). In the rest of this section we illustrate the pairs of in-script and out-script which are considered standard by the Bitcoin network [39]. In Section 3 we will then show how these scripts are commonly used for embedding metadata.
We use the typewriter font for denoting opcodes (e.g., OP_CHECKSIG), and italic for denoting bitstrings (e.g., sig). In Bitcoin scripts, these bitstrings are always preceded by a suitable OP_PUSHDATA opcode, which pushes the bitstring onto the stack. For the sake of simplicity, hereafter we omit these OP_PUSHDATA opcodes.

\subsubsection{Pay to public key (P2PK)}

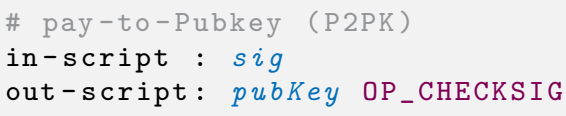

This pair of scripts implements the signature verification function outlined in Section 2.1. The evaluation of the output script starts with sig on top of the stack, and then proceeds by pushing also pubKey. The opcode OP_CHECKSIG performs the signature verification, popping the two top elements of the stack, and evaluating to true if the verification succeeds.

\subsubsection{Pay to public key hash (P2PKH)}

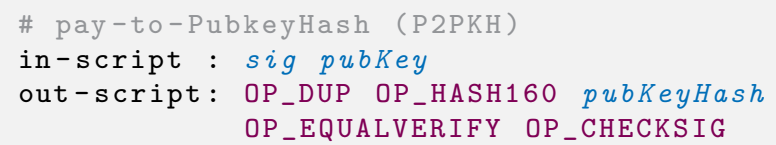

This pair of scripts performs signature verification, similarly to the previous pair. The main difference with respect to $\mathrm{P} 2 \mathrm{PK}$ is that the output script now contains the double hash of a public key, rather than the public key itself.

More in detail, the in-script contains a signature sig and a public key pubKey. The evaluation of the output script starts with sig and pubKey on the stack. The opcode OP_DUP duplicates the top element of the stack (i.e., pubKey), and OP_HASH160 replaces the top element with its double hash. Then, pubKeyHash (the double hash of a key) is pushed into the stack. The opcode OP_EQUALVERIFY checks if the two top elements of the stack are equal: if so, they are popped, otherwise the script fails. Finally, OP_CHECKSIG performs the signature verification.

\subsubsection{Multi-signature}

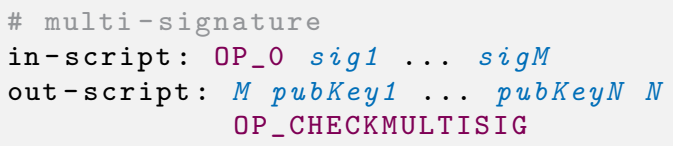


This pair of scripts performs a multi-signature verification: the output can be redeemed if the in-script provides $M$ signatures verified against $N$ public keys, where $M \leq N$. The opcode OP_CHECKMULTISIG tries to verify the last signature with the last public key. If they match, it proceeds to verify the previous signature in the sequence, otherwise it tries to verify the signature with the previous key. Notably, OP_CHECKMULTISIG uses each key only once, therefore the order of the signatures in the in-script matters.

\subsubsection{Pay to script hash (P2SH)}

\# pay-to-ScriptHash (P2SH)

in-script : v1 ... vN bitstring

out-script : OP_HASH160 hash OP_EQUAL

In this pair, firstly the out-script is evaluated with bitstring on top of the stack. The script checks that the hash of bitstring is equal to hash. If so, the script obtained by interpreting bitstring as a sequence of opcodes is executed (on the parameters $v 1 \ldots v N$ ). Summing up, the output can be redeemed if the in-script of the redeeming transaction provides a script whose hash coincides with the hash contained in out-script, and whose evaluation (on the parameters $v 1 \ldots v N$ ) yields true.

\subsubsection{OP_RETURN}

\# op_return

out-script: OP_RETURN bitstring

An out-script containing OP_RETURN always evaluates to false, regardless of the value of the bitstring. Therefore the corresponding output is unspendable, and it can be safely removed from the UTXO set.

Currently, standard transactions can have only one occurrence of OP_RETURN: more precisely, if a transaction has more than one out-script with OP_RETURN, or an out-script with more than one OP_RETURN, or an OP_RETURN with more than one OP_PUSHDATA, then the transaction is not standard.

\section{Embedding metadata in the blockchain}

In Sections 3.1 to 3.7 we illustrate various techniques (as far as we know, all those used in practice) to embed metadata in the blockchain. In Section 3.8 we show how to split large pieces of metadata into smaller pieces that can be distributed among sets of transactions. In Section 3.9 we discuss how to commit to specific values without explicitly writing them in the blockchain. Section 3.10 gives some statistics on embedding techniques.

\subsection{Value field}

Transaction outputs specify the amount of Satoshis to send through the value field, of 8 bytes size. A first way to encode a message $m$ in the blockchain is to build a transaction with an output whose value is the number that represents $m$. For instance, the BitcoinTimestamp protocol (see Table 5) exploits this method for saving a SHA256 hash. The hash is first split into 16 pieces, which are then translated into amounts of Satoshis. Finally, the protocol builds a transaction containing an output for each amount (e.g. see rows 1-2 of Table 1).

Although users can easily recover the moved funds (e.g. by specifying their own address as receiver), the disadvantage of this technique is that it requires to own at least the amount of Satoshis needed to represent $m$.

\subsection{Input sequence}

Some users exploited the 4 bytes in the sequence_no field for appending their own metadata. Although this technique does not have negative effects on the Bitcoin system, as far as we know no protocols use this technique (as shown in Table 5). We conjecture that protocols rely on other techniques because 4 bytes are not enough for implementing any relevant use case (see Section 4 for a description of protocols and use cases).

\subsection{Pay to public key / Pay to public key hash}

In P2PK (Section 2.2.1) the output script specifies the recipient of some bitcoins through a public key, encoded in 65 bytes (or 33 bytes when the key is compressed). Similarly, P2PKH (Section 2.2.2) uses the hash of the key, which is encoded in 20 bytes.

Users can embed an arbitrary message $m$ (of suitable size) in the P2PK or P2PKH script of some output of a transaction $T$, by writing $m$ in place of the expected key or hash. Assuming that Bitcoin uses secure signatures and collision-resistant hash functions, it is computationally unfeasible, in general, to craft a transaction which redeems such output of $T$.

A downside of this approach is that these unspendable outputs are indistinguishable from the spendable ones: actually, a Bitcoin node has no way of knowing whether or not a user exists who possesses the needed hash preimage (nor does it know that the data was never intended to represent an address in the first place). 


\begin{tabular}{|c|l|l|}
\hline$\#$ & Technique & Transaction identifier / Bitcoin address \\
\hline \hline 1 & \multirow{2}{*}{ Value } & f6f89da0b22ca49233197e072a39554147b55755be0c7cdf139ad33cc973ec46 \\
2 & & 49a130ce4255fc91061c3d1170cbc256f51ed671256df837500d59183cfdd64f \\
\hline 3 & OP_RETURN & d84f8cf06829c7202038731e5444411adc63a6d4cbf8d4361b86698abad3a68a \\
\hline 4 & & 1ponziUjuCVdB167ZmTWH48AURW1vE64q \\
5 & & 1CounterpartyXXXXXXXXXXXXXXXUWLpV \\
6 & & 72162e9224dbadefb84834046ee8b4706af77f57fa4e8fd5aaf3255abf516807 \\
7 & & 1WeRe3jh9XiaAyabyiE2Mz4v8bbcB52Gy \\
8 & Vanity Address & 1FineoW99TYAAZuRSkbZLrx65iTXELqHhv \\
9 & & 18chaNzLXvAbYvkad7MH2LNrQmBzeXbWLo \\
10 & & 1PoStJBYu49Ezqcwh1VeMWZgRopwcYwksY \\
11 & & 1FAke1neYErMQLebVPYBAToTLvafr5ZPF6 \\
\hline 12 & Coinbase & 4a5e1e4baab89f3a32518a88c31bc87f618f76673e2cc77ab2127b7afdeda33b \\
\hline 13 & P2PKH & 5970ae129d1141663bd5e441a1555c16fb1c0586dd05f40c1db3d3e81218ee41 \\
\hline 14 & P2SH & 1e47936f37e71b98e8bafe51ddc902d59c1318bc556329ba4ab1996981785292 \\
\hline
\end{tabular}

Table 1: Some transactions and addresses containing metadata.

As a result, the nodes of the Bitcoin network must keep these transactions in their UTXO set indefinitely. Since the UTXO set is usually stored in RAM for efficiency concerns [52], the bloating of the UTXO set negatively affects the memory consumption of nodes [32].

\subsection{Pay to script hash}

By using P2SH scripts (Section 2.2.4), metadata can be embedded in various ways. Similarly to the P2PKH technique (Section 3.3), one can embed metadata in the output script, in place of the hash. An alternative technique is to embed metadata in the input script, pushing them onto the stack with the OP_PUSHDATA instruction, and immediately afterwards removing them with OP DROP. As long as the script completes its execution successfully and there is some nonzero value on the stack after completion, the transaction is valid [10]: indeed, there is no rule specifying that the data accumulated on the stack during the script execution must be cleared. Stack items that are below the topmost item at the end of execution are simply ignored. For instance, consider the following scripts:

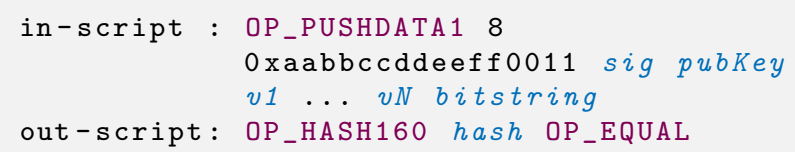

First, the in-script and the out-script are concatenated; the result is a common $\mathrm{P} 2 \mathrm{SH}$ script preceded by an OP_PUSHDATA1. The evaluation starts by pushing Oxaabbccddeeff0011 onto the stack. This is done through OP_PUSHDATA1, where the trailing 1 indicates that the next 1 byte contains the number of bytes to be pushed onto the stack (in the snippet above, 8 bytes). At the end of the execution, the top item on the stack is true, resulting from the OP_EQUAL. Underneath this value there is the metadata $0 x a a b b c c d d e e f f 0011$.
Note that transactions that make use of ignored OP_PUSHDATA for embedding metadata do not bloat the UTXO set: indeed, their outputs can be spent by valid addresses, because they do not need to overwrite the address fields in the transaction scripts. The work [26] provides further details on the $\mathrm{P} 2 \mathrm{SH}$ technique.

\subsection{OP_RETURN}

Standard OP_RETURN transactions allows to store up to 80 bytes of arbitrary data (see e.g. row 3 of Table 1 ). An out-script containing OP_RETURN always evaluates to false, hence the output is unspendable, and its transaction can be safely removed from the UTXO set. In this way, OP_RETURN overcomes the UTXO consumption issue highlighted in Section 3.3.

\subsection{Vanity address}

Bitcoin addresses are the hash of ECDSA public keys. By a brute force search during the generation of the key pair, it is possible to obtain a so-called vanity address, where a few bytes are equal to a given string (see e.g. row 4 of Table 1$)^{2}$. One can embed these bytes as metadata in transactions, e.g. by using the vanity address in P2PK scripts.

Although, in theory, the maximum size of the metadata corresponds to the size of an address (20 bytes), in practice this technique is practical only for metadata of the size of a few bytes. Longer metadata can be distributed among different vanity addresses; for instance, the transaction at row 6 of Table 1 transfers bitcoins from 5 vanity addresses (displayed at rows 7-11). By concatenating the first four characters (but the leading

2 Open-source tools like Vanitygen generate vanity addresses with user-defined patterns. 
1) of these addresses, we read the plain English words: "We're fine, 8chan post fake".

A different use of vanity addresses is when all the bytes of the address are fixed, like e.g. at row 5 of Table 1 . In this case, it is plausible that the corresponding private key is not known to anybody, so a P2PK output using such address cannot be spent. This fact is exploited to implement "Proof-of-Burn" protocols on top of Bitcoin (like e.g. in Counterparty): in these protocols, users receive some tokens in exchange for sending some bitcoins to an unspendable address.

\subsection{Coinbase transaction}

Miners specify how to redeem the reward for the mined block (and the fees of its transactions) through the first transaction of the block. This transaction does not have an input script, and it contains a field called coinbase, that miners usually fill in with metadata. The coinbase data size is between 2 and 100 bytes. Nevertheless, after block 227,835 the available space is reduced, since the Bitcoin Improvement Proposal 34 (BIP 0034) [27] requires the first bytes of the coinbase field to store the block height index.

Usually, the coinbase field is used by miners for identifying the mining pool, or for voting BIPs (for instance, when they voted to support either the BIP 0016 or BIP 0017 [28]). The most famous message embedded by using this technique is included in the genesis block (see e.g. row 12 of Table 1): "The Times 03/Jan/2009 Chancellor on brink of second bailout for banks".

\subsection{Distributing metadata}

The techniques discussed in Sections 3.1 to 3.7 embed metadata within a single field of a single transaction. Below we describe three techniques that allow to split metadata among multiple fields and transactions.

Multisignature. This technique uses the multisignature script introduced in Section 2.2.3. Given $N-1$ pieces of metadata $v 2, \ldots, v N$, the scripts are the following:

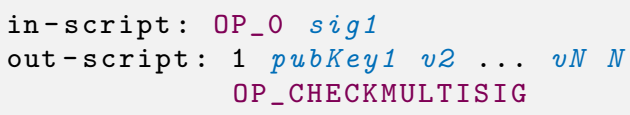

This implements a 1-of- $N$ multisignature: namely, only one signature (sig1) needs to be verified against some of the $N$ public keys (pubKey1). The other "public keys" in the out-script - actually, the $N-1$ pieces of metadata - are irrelevant for the execution of the script.

Note that this technique bloats the UTXO set only until the transaction is redeemed.
Multiple inputs / outputs. Although Bitcoin imposes a hard limit of $10 \mathrm{~K}$ bytes on the size of a single script [9], there is no limit to the number of inputs or outputs a transaction may contain. Hence, metadata bigger than $10 \mathrm{~K}$ bytes can be split into smaller chunks, and distributed among many inputs or outputs within a single transaction. To ensure that the original data can be reconstructed from these fragments, one needs to fix an encoding. The simplest one is to store the chunks of metadata sequentially in $N$ output scripts. Another common encoding, used e.g. by the BIT-COMM protocol, uses the amount of bitcoins transferred by each output to order the chunks (see e.g. row 13 of Table 1 ). Transactions using this technique may or may not bloat the UTXO set - that is determined by the structure of the individual transaction outputs.

Transaction chains. The previous techniques store metadata within a single transaction. However, this is not always ideal, or even possible. For instance:

- If the size of metadata exceeds the maximum block size, the transaction containing the metadata will be rejected by the network. ${ }^{3}$

- Large transactions require large fees. Even though, in theory, one can send a transaction with zero fee, in practice a transaction with no fee (or a low fee) is unlikely to be mined. Depending on current fee market dynamics, it may be more cost-effective to split the metadata across multiple transactions.

- Transactions greater than a certain size, or which contain more than one OP_RETURN, are considered non-standard, with the consequence that most nodes refuse to relay them. This limit has varied over time, and is now replaced by the concept of "transaction weight" which is similar, but accounts for Segregated Witness data in a different manner. ${ }^{4}$

Due to these considerations, metadata are often split into sets of transactions. A common technique is to connect transactions containing related data in a chain structure. When building a transaction chain, one of the techniques from Sections 3.1 to 3.7 is chosen for encoding data into each individual transaction. Then, a spendable transaction output is added to each transaction, to be redeemed by the subsequent transaction in the chain. The output must be spendable by an address over which the user embedding the data has control.

\footnotetext{
3 See github.com/bitcoin/.../src/main.cpp\#L829.

4 See github.com/bitcoin/bitcoin/.../src/main.h\#L56, and github.com/bitcoin/bitcoin/.../src/main.cpp\#L644-L648.
} 


\subsection{Embedding vs. committing metadata}

Several Bitcoin-based protocols notarize documents by embedding their hash on the blockchain. There are alternative techniques, e.g. pay-to-contract [43] and sign-to-contract ${ }^{5}$, which allow one to commit to a hash without actually embedding it into a transaction. For instance, Btproof applies RIPE160 to the hash, to obtain a Bitcoin address; similarly, Originstamp daily aggregates hashes into a seed, hashes it into a private key, and then derives from it the corresponding public key and address. Both protocols pay a small fee to the generated address in order to publish it in the blockchain. ContractHashTool exploits elliptic curves for building an in-script that cryptographically commits to a given hash, without embedding the hash itself. Unless a protocol keeps a public track of its transactions (like, e.g., Originstamp), these techniques prevent external observers from inferring any metadata.

\subsection{Statistics on embedding techniques}

Table 2 shows the amount of metadata embedded with each technique. The leftmost column groups the techniques in three categories: the Single category contains the techniques which embed the whole piece of metadata into a single chunk; Multi contains the techniques which split an element into multiple chunks, but store all the pieces into a single transaction; finally, Chains gathers the techniques which spread pieces across multiple transactions. The third and fourth columns show the size (in bytes) of the field storing metadata, and where the field is located. The fifth column lists the techniques bloating the UTXO. The sixth column displays the date in which first chunk of metadata appears, the seventh one shows the number of times a technique has been used ${ }^{6}$, and last two columns show the total and average size of metadata embedded.

In the first 480,000 blocks we count 4,582,661 chunks of metadata, for a total size of $\sim 99 \mathrm{MB}$. Note that, since the chunks of the Chains type are a subset of those in the other categories, and the chunks in Multi are a subset of those in Single, to avoid counting the same piece of metadata multiple times, the totals only consider the values of the Single type. This number of chunks is a good indicator of the total number of transactions with metadata, since the fraction of transactions produced by Multi techniques is negligible. We observe that $\sim 75 \%$ of the space used by metadata is due

\footnotetext{
5 See bitcointalk.org/index.php?topic=915828.msg10056796

6 Elements of type Single are chunks; Multi elements are transactions; Chains elements are chains of transactions.
}

to OP_RETURN transactions. The average size of transactions chains is higher than other methods, since this technique is used for embedding images and archives.

\section{Analysis of Bitcoin metadata}

In this section we present our techniques to parse metadata and reconstruct the original content. Then, we categorize the reconstructed items, and we measure them.

\subsection{Collecting metadata}

One of the most effective techniques for recognising chunks of metadata is to search strings for "suspicious" byte patterns. For example, long strings of contiguous ASCII characters are unlikely to occur in regular transaction data; similarly, the probability of finding specific bitstrings, like the Gzip header 0x1f9d9070, is extremely low. Finding such a bitstring is a trigger for further investigation. We employed several types of these searches, which we discuss below.

Frequency analysis. The GNU strings utility ${ }^{7}$ takes a data source as input, and yields as output all of the ASCII plaintext characters found in that source. It provides a flag for filtering out strings of contiguous ASCII characters under a given length. It is possible to run strings directly on Bitcoin Core's .dat files, but care must be taken when tuning the filter. Obviously, too low a threshold will yield a huge number of false positives. On the other hand, due to the way inputs and outputs are encoded in transaction data, too high a threshold eliminates plaintext that has been split across multiple transactions or transaction scripts.

While this approach is quite simple, some of the data that we encountered - particularly, the conversations and code embedded into the blockchain by Peter Todd (one of the Bitcoin Core developers) - mention that they are specifically intended to be discovered and extracted via this method. For example, Todd's plaintext uploader is a Python script stored in the blockchain (see row 14 of Table 1). It describes itself as a tool that can "publish text in the blockchain, suitably padded for easy recovery with strings". 8 The tool appears to have been used to upload its own source code to the blockchain. Peter Todd's tool takes a text file as input, and uses the P2SH ignored OP_PUSHDATA technique (see Section 3.4) to embed the contents of the file into the

\footnotetext{
7 man7.org/linux/man-pages/man1/strings.1.html

8 github.com/petertodd/python-bitcoinlib/blob/ master/examples/publish-text.py\#L48
} 


\begin{tabular}{|c|c|c|c|c|c|c|c|c|}
\hline Type & Technique & $\begin{array}{c}\text { Field } \\
\text { size }\end{array}$ & Hosted in & $\begin{array}{c}\text { UTXO } \\
\text { Bloating }\end{array}$ & 1st item & \# items & Tot. size & Avg. size \\
\hline \multirow{8}{*}{ Single } & Value & 8 & Tx output & No & $\mathrm{N} / \mathrm{A}$ & $\mathrm{N} / \mathrm{A}$ & N/A & $\mathrm{N} / \mathrm{A}$ \\
\hline & Input Sequence & 4 & Tx input & No & $2011 / 02 / 25$ & $1,305,372$ & $5,221,488$ & 4 \\
\hline & P2PK-P2PKH & $20,33,65$ & Script & Yes & $2013 / 03 / 16$ & 66,762 & $1,335,240$ & 20 \\
\hline & P2SH & 520 & Script & Yes & $2013 / 04 / 10$ & 1,578 & 31,560 & 20 \\
\hline & OP_RETURN & 80 & Script & No & 2014/03/12 & $2,903,186$ & $76,700,965$ & 26 \\
\hline & Vanity Address & 20 & Script & No & $\mathrm{N} / \mathrm{A}$ & $\mathrm{N} / \mathrm{A}$ & $\mathrm{N} / \mathrm{A}$ & $\mathrm{N} / \mathrm{A}$ \\
\hline & Coinbase & $2-100$ & Tx input & No & $2009 / 01 / 03$ & 305,763 & $18,442,641$ & 60.3 \\
\hline & Total & - & - & - & $2009 / 01 / 03$ & $4,582,661$ & $101,731,894$ & 22 \\
\hline \multirow{2}{*}{ Multi } & Multi-signature & Variable & Script & Transient & $2013 / 04 / 06$ & 15,067 & $2,926,590$ & 194 \\
\hline & Multi-in/out & Variable & Variable & Variable & $2013 / 03 / 16$ & 529 & $4,437,616$ & 8,389 \\
\hline Chains & Tx chains & Variable & Variable & Variable & $2013 / 04 / 06$ & 60 & $3,470,870$ & 57,848 \\
\hline
\end{tabular}

Table 2: Statistics about embedding techniques (sizes are in bytes).

input scripts of a single transaction. The reason this encoding lends well to strings-based extraction is that it allows large amounts of arbitrary data to be stored with minimal interruption by non-ASCII bytes. Input scripts are stored contiguously in transaction data, meaning that the only necessary interruptions will be the minimal set of Bitcoin script opcodes required to ensure that the transaction is considered valid by the network.

Compared to the other methods, strings-based extraction offers the lowest barrier-to-entry. Thus, users encoding large quantities of plaintext data that are intended to be easily discoverable should make note of encoding methods that lend well to this technique.

File signature. Many file formats require the inclusion of specific bytestrings that are common to all files of a given format. For example, many JPEG images begin with the bytestring 0xffd8ffe000104a464946000101. Similarly, ASCII-armored PGP messages begin with -----BEGIN PGP MESSAGE-----. These bytestrings often occur in the header or footer of the file, although there are formats that place them elsewhere. The probability of finding such bytestrings in Bitcoin blocks is exceedingly low, and as such, they provide a useful indicator of embedded data.

We used several tools to detect file signatures present in Bitcoin transactions:

binwalk is an extensible tool for discovering valid files embedded into other data [41]. It provides a language for defining file signatures, as well as a large database of pre-defined signatures for common file formats. It also has the ability to carve detected files out of the surrounding binary data. One can produce a number of valid results simply by running binwalk on the Bitcoin Core .dat files. However, since the tool is unaware of the Bitcoin block format, it is only suitable for recovering files embedded into a single transaction script. binary-grep searches a collection of input files for a single bytestring specified by the user [37]. It outputs the byte offsets of any matches, and has a simple carving function.

local-blockchain-parser provides a grep command that, unlike binwalk and binary-grep, is aware of the Bitcoin block format [38], skipping the parts of the transaction that cannot embed metadata. For each match, it outputs the block and transaction hashes, script type (input/output), and byte offset.

One of the most successful workflows we discovered for recovering binary files based on file signatures was the following. (i) We ran binwalk and/or binary-grep on a .dat file, making note of any results that appeared to be true positives. (ii) If there were promising results, we then ran the binary-grep subcommand of local-blockchain-parser on that .dat file, obtaining the transaction hashes where those results were found. (iii) For each resulting transaction, we manually inspected the transaction graph around it. If it appeared to be an isolated transaction, we ran the tx-info subcommand of local-blockchain-parser. If it appeared to be a part of a chain, we ran the tx-chain subcommand instead. (iv) We inspected the binary output from the previous step, performing manual carving where necessary, and we attempted to ascertain the validity of the results by opening them with applications appropriate to their file type.

Protocol Identifier. Many protocols mark their metadata by writing a specific string in the first few bytes of each chunk, but the exact number of bytes may vary from protocol to protocol. In Section 5 we take advantage of this for associating metadata to protocols. Furthermore, since protocols give a detailed description of the format of the elements produced, in Section 4.2 we distinguish different types of metadata and classify them. Hence, in order to associate metadata to protocols we: (i) search the web for known associations 
between identifiers and protocols; (ii) we accordingly classify strings beginning with one of the identifiers obtained. In more details, in the first step we query Google to obtain public identifier/protocol bindings. For instance, since several protocols use the OP_RETURN technique, we execute the query "Bitcoin OP_RETURN", that returns $\sim 26,500$ results, and we manually inspect the first few pages of them. Note that a protocol can be associated with more than one identifier (e.g., Stampery, Blockstore), or even do not have any identifier. In this way we obtain 45 protocols associated to 39 identifiers; further, we find several protocols that do not use any identifier (e.g., Diploma, Chainpoint). We also distinguish the main types of metadata produced by protocols (e.g. Text, Hash and Record). The second step is performed by our tool: it associates chunks of metadata to a protocol. The full list of protocols discovered is shown in Table 5; identifiers are listed in Table 4.

Transaction chains. Although all spent transaction outputs in the Bitcoin blockchain naturally form a chain structure, identifying chains containing embedded metadata is not entirely straightforward. A transaction may have certain "giveaway" characteristics that suggest the presence of a chain containing data, such as:

1. One or more unspendable outputs (i.e., OP_RETURN outputs), plus a single spent output. The unspendable output(s) would contain data, while the spent output would be used to continue the chain.

2. One or more unspent outputs (possibly used for a P2PKH embedding), plus a single spent output. The unspent output(s) would contain data, while the spent output would be used to continue the chain.

3. The unspent outputs, if any, contain a tiny amount of Satoshis (such outputs are also known as dust). Except for the Bit-Comm protocol, which uses output values to order the data in the output scripts, the funds included into outputs that can never be spent are effectively "burnt", and add no information to the embedded data. This disincentivizes the embedder from including any more value than is strictly necessary to create a valid transaction.

4. The spent output contains a relatively large amount of bitcoins, used to fund further dust outputs in subsequent links in the chain.

5. Preceding or subsequent transactions share a similar structure with the transaction in question. Many of the transaction chains we found appeared to have been constructed with the help of software (e.g. the Python source we extracted). The software we found tends to create strings of transactions sharing a similar format. While it is altogether possible to em- bed data into chains of dissimilar transactions, they would be difficult to find and complex to decode.

These are helpful clues, but not definitive criteria. In fact, there are many other types of transactions which possess the characteristics described above. For example, payouts from mining pools and Bitcoin casinos often send small amounts of bitcoins to many users at once. These payout transactions are often constructed algorithmically (according to some set of "threshold" rules intended to minimize the impact of the fee on the payout), meaning that preceding and following transactions share a similar structure.

Therefore, it is generally necessary to have some understanding of the embedded data in order to determine whether a given chain is of interest. If a transaction contains a file signature for a file type that is unlikely to fit into the data provided by that transaction, it warrants further investigation.

Extraction of data from transaction chains is relatively easy when using the local-blockchain-parser utility. This utility has a tx-chain subcommand that takes a single transaction hash and crawls backwards and forwards through the transaction graph, collecting data from the transaction scripts. This data is filtered and permuted to account for the various ways in which transaction chains are constructed. Finally, the data from each transaction are concatenated in the order that they appear in the chain. This process yields a collection of binary files corresponding to the different ways in which data can be embedded into a chain.

\subsection{Types of metadata}

We associate the successfully reconstructed data items to one of the following types:

Text Users have embedded a significant amount of text, since the very first message by Satoshi Nakamoto. This includes several birthday wishes, love statements, prayers, greetings, developer conversations, and magnet links. Besides user messages, miners usually embed in coinbase transactions messages for Bitcoin-related purposes, to identify their blocks, vote on proposals, announce what features they support. We have also identified two pdf documents: "Bitcoin: a peer-to-peer electronic cash system" [48], and "The first collision for full SHA-1" [51].

Hash Many users notarize the ownership of documents by embedding their hash on the blockchain (embedding the whole document would be too expensive, because of the required transaction fees). Some protocols notarize several documents with a single piece of metadata; this could be the hash of the sequence 
of document hashes, or the root of the Merkle tree of the document hashes.

Financial record A common application of the Bitcoin blockchain is to record the ownership and exchange of digital or physical assets. These assets are represented as tokens, and users are identified by their Bitcoin addresses.

Copyright Copyright records are produced by protocols which act as marketplaces where artists publish and sell their files to other users.

Script Developers have embedded in the blockchain several scripts. We have found some Python scripts (e.g., the Satoshi uploader, Satoshi downloader, and Cryptograffiti uploader), Bash scripts (e.g., Password script, and OpenSSL encoder), and also some games (e.g., LinPyro, Bong ball and Lucifer).

Image The blockchain contains some small images, usually spread across chains of transactions. We have found various file formats (PNG, JPEG, and GIF).

Archive This type includes compressed archives, like e.g. the WikiLeaks Cablegate gzipped archive.

\subsection{Statistics on types of metadata}

Table 3 shows some statistics about the type of metadata we reconstructed. The second column indicates the day in which the first piece of metadata of the corresponding type appeared in the blockchain. Next, we show the total number of elements found, followed by their total size in bytes, and their average size.

Note that the total size of reconstructed metadata is less than the 101,731,894 reported in Table 2: this is because Table 2 also includes bitstrings that we did not manage to decode. For instance, the bytes embedded with OP_RETURN are always considered in Table 2, but they appear in Table 3 only if we are also able to recognize their type (e.g., because their prefix reveals which protocol has produced them, among those in Table 4). From the rightmost column we see that the average size of scripts, images and archives exceeds the maximum size of Bitcoin scripts; hence, these metadata are embedded through "Multi-in/out" or "Tx chains" techniques. Finally, note that although the first financial record appeared only in May 2014, this type of metadata now constitutes $\sim 70 \%$ of the reconstructed elements, and it uses the majority of the space.

\section{Analysis of Bitcoin-based protocols}

In this section we focus on protocols which embed metadata on the Bitcoin blockchain. We first propose a rough taxonomy of protocols, which categorize them according to the application domain. Then, considering the collection of protocols reported in a previous paper [34], we perform several analyses on their usage of metadata.

\subsection{Types of protocols}

Our taxonomy classifies protocols in five categories:

Financial includes protocols that manage assets, e.g. for certifying their ownership, endorsing their value, and keeping track of trades. Metadata in these transactions are used to specify the value of the asset, the amount of the asset transferred, the new owner, etc.

Notary includes protocols that certify the ownership and timestamp of documents. These protocols allow users to publish the hash of a document in a transaction, thus proving its existence and integrity. Since the transaction is signed with a private key, users can also certify the ownership of the document.

DRM includes protocols for declaring access rights and copyrights on digital art documents, like e.g. images or audio files.

Message groups protocols which record text messages. Subchain gathers protocols which construct transaction chains to record execution traces of third-party smart contracts.

We now briefly comment this taxonomy. Although Notary and DRM protocols have the same overall goal - certifying the ownership of documents - they have some relevant differences. First, Notary protocols do not usually require the original document (yet, they ask that the document hash is provided by the owner); further, their goal can be fulfilled also when their frontend is no longer online. Conversely, DRM protocols usually need to gather user documents, and have complex front-ends to enable further interactions with users (e.g., they often play the role of broker between media producers and consumers). The ordering of metadata embedded by Notary, DRM and Message protocols is immaterial; instead, different orderings in Financial and Subchain protocols usually imply different system states. Indeed, transactions used by Financial protocols are analogous to Bitcoin transactions, except that they transfer tokens instead of bitcoins; depending on the current balance of tokens, appending a transaction may result in a state update, or even leave the state unchanged (e.g., if an attacker attempts to sell assets that she does not currently own). Subchain protocols share the same mechanism, but they generalize token exchange to more complex computations, like those arising from the execution of smart contracts (e.g., in the RSK platform). 


\begin{tabular}{|c|c|r|r|r|}
\hline Type & 1st item & \# items & Tot. size & Avg. size \\
\hline \hline Text & $2009 / 01 / 03$ & 309,894 & $18,811,329$ & 61 \\
Hash & $2013 / 12 / 18$ & 200,832 & $7,617,392$ & 38 \\
Financial record & $2014 / 05 / 03$ & $1,430,071$ & $37,699,809$ & 26,36 \\
Copyright & $2014 / 12 / 19$ & 116,406 & $3,503,170$ & 30 \\
Script & $2013 / 04 / 06$ & 10 & 138,149 & 13,815 \\
Image & $2013 / 03 / 17$ & 108 & $1,523,529$ & 14,107 \\
Archive & $2013 / 04 / 06$ & 12 & $2,838,760$ & 236,563 \\
\hline TOTAL & $\mathbf{2 0 0 9 / 0 1 / 0 3}$ & $\mathbf{2 , 0 5 4 , 5 7 5}$ & $\mathbf{7 2 , 1 3 2 , 1 3 8}$ & $\mathbf{3 5}$ \\
\hline
\end{tabular}

Table 3: Statistics on types of metadata (sizes are in bytes).

\subsection{Statistics on Bitcoin-based protocols}

Table 5 shows some detailed statistics about protocols. The first and second columns indicate, respectively, the protocol type and name. We use an additional type, called Empty, to gather the transactions which use OP_RETURN without embedding any metadata. The third and fourth columns show the type of metadata and the embedding technique. The fifth column shows when the protocol generated the first chunk of metadata; since transactions do not carry a timestamp, to this purpose we use the timestamp of the enclosing block. The next two columns count the total number of elements produced by a protocol, and the total size (in bytes) of the embedded metadata (net of script instructions and other transaction fields). The rightmost column shows the average size of the metadata.

We were able to associate to protocols $\sim 53.7 \mathrm{MB}$ of metadata, which is quite less than the total amount extracted $(\sim 99 \mathrm{MB})$. This difference has various motivations. First, users often embed metadata not related to any protocol; for instance, this is the case for several images and text messages. Second, several protocols make it impossible, for an external observer, to recognize their chunks of metadata (unlike the protocols in Table 4, which append an identifier to the metadata): indeed, we have discovered 19 protocols that embed metadata without any identifier. Finally, our list of protocols may be incomplete, so if some other protocols embed metadata with OP_RETURN, we count their items but we can not classify them. We note a relevant component of Empty transactions ( $\sim 10 \%$ of the total OP_RETURN transactions), which use OP_RETURN without any data attached, so they are not associated to any protocol. We evaluate that $\sim 96 \%$ of these transactions are related to the peaks, discussed later on in Section 6.4. The fifth column of Table 5 suggests that, originally, the protocols were of Financial and Notary type, while the other use cases were introduced subsequently (indeed, the others types were not inhabited before the end of 2014).

\begin{tabular}{|c|c|c|}
\hline Type & Protocol & Identifiers \\
\hline \hline \multirow{5}{*}{ Financial } & Colu & CC \\
& CoinSpark & SPK \\
& OpenAssets & OA \\
& Omni & omni \\
& Openchain & OC \\
& Helperbit & HB \\
\hline \multirow{5}{*}{ Notary } & Counterparty & CNTRPRTY \\
& Factom & Factom!!, FACTOM00, Fa, FA \\
& Stampery & S1, S2, S3, S4, S5, S6 \\
& Proof of Existence & DOCPROOF \\
& Blocksign & BS \\
& CryptoCopyright & CryptoTests-, CryptoProof- \\
& Stampd & STAMPD\#\# \\
& BitProof & BITPROOF \\
& ProveBit & ProveBit \\
& Remembr & RMBd, RMBe \\
& LaPreuve & ORIGMY \\
& Nicosia & LaPreuve \\
& SmartBit & UNicDC \\
& Notary & SB.D \\
\hline \multirow{2}{*}{ DRM } & Monegraph & Notary \\
& Blockai & MG \\
& Ascribe & Ox1f00 \\
\hline \multirow{2}{*}{ Message } & Eternity Wall & ASCRIBE \\
& BitAlias & EW \\
\hline Subchain & Blockstore & BALI \\
\hline
\end{tabular}

Table 4: Protocol identifiers. Counterparty metadata must be first deobfuscated with ARC4 encryption, using the transaction identifier of the first unspent transaction output as the encryption key.

From Table 5 we see that the large majority of protocols use the OP_RETURN technique. Focussing on the metadata embedded with this technique, Figure 2 displays how metadata are distributed into the protocol types, and Figure 3 shows the temporal evolution of their usage, in terms of the number of metadata items published per week. Comparing Table 5 with Figure 2 we see that although most protocols are Notary, their transactions are a fraction of those produced by Financial protocols. 


\begin{tabular}{|c|c|c|c|c|c|c|c|}
\hline Type & Protocol & Metadata & Technique & 1st item & \# items & Tot. size & Avg. size \\
\hline \multirow{10}{*}{ Financial } & $\overline{C \text { Colu }}$ & Financial record & OP_RETURN & $2015 / 07 / 09$ & 244,411 & $4,425,702$ & 18 \\
\hline & CoinSpark & Financial record & OP_RETURN & $2014 / 07 / 02$ & 28,120 & 960,664 & 34 \\
\hline & OpenAssets & Financial record & OP_RETURN & $2014 / 05 / 03$ & 207,132 & $3,255,499$ & 16 \\
\hline & Omni & Financial record & OP_RETURN & $2015 / 08 / 10$ & 311,605 & $6,249,883$ & 20 \\
\hline & Openchain & Hash & OP_RETURN & $2015 / 10 / 21$ & 2,758 & 115,283 & 42 \\
\hline & Helperbit & Financial record & OP_RETURN & $2015 / 09 / 18$ & 33 & 1,251 & 38 \\
\hline & & & OP_RETURN & $2014 / 06 / 16$ & 636,012 & $22,806,810$ & 36 \\
\hline & Counterparty & Financial record & P2PKH & $\mathrm{N} / \mathrm{A}$ & $\mathrm{N} / \mathrm{A}$ & $\mathrm{N} / \mathrm{A}$ & $\mathrm{N} / \mathrm{A}$ \\
\hline & & & Multi-signature & $\mathrm{N} / \mathrm{A}$ & $\mathrm{N} / \mathrm{A}$ & $\mathrm{N} / \mathrm{A}$ & $\mathrm{N} / \mathrm{A}$ \\
\hline & Total & - & - & $2014 / 06 / 16$ & $1,430,071$ & $37,815,092$ & 26 \\
\hline \multirow{25}{*}{ Notary } & Factom & Merkle root & OP_RETURN & $2014 / 04 / 11$ & 105,188 & $4,207,262$ & 40 \\
\hline & Stampery & Merkle root, Hash & OP_RETURN & $2015 / 03 / 09$ & 74,887 & $2,648,102$ & 35 \\
\hline & Proof of Existence & Hash & OP_RETURN & $2014 / 04 / 21$ & 5,464 & 218,513 & 40 \\
\hline & Blocksign & Hash & OP_RETURN & $2014 / 08 / 04$ & 1,477 & 55,676 & 38 \\
\hline & CryptoCopyright & Hash & OP_RETURN & $2014 / 08 / 02$ & 46 & 1,840 & 40 \\
\hline & Stampd & Hash & OP_RETURN & $2015 / 01 / 03$ & 562 & 22,427 & 40 \\
\hline & BitProof & Hash & OP_RETURN & $2015 / 02 / 25$ & 770 & 30,800 & 40 \\
\hline & ProveBit & Hash & OP_RETURN & $2015 / 04 / 05$ & 57 & 2,280 & 40 \\
\hline & Remembr & Hash & OP_RETURN & $2015 / 08 / 25$ & 28 & 1,128 & 40 \\
\hline & OriginalMy & Hash & OP_RETURN & $2015 / 07 / 12$ & 126 & 4,788 & 38 \\
\hline & LaPreuve & Hash & OP_RETURN & $2014 / 12 / 07$ & 68 & 2,663 & 39 \\
\hline & Nicosia & Hash of hashes & OP_RETURN & $2014 / 09 / 12$ & 24 & 840 & 35 \\
\hline & SmartBit & Merkle root & OP_RETURN & $2015 / 11 / 24$ & 8,472 & 304,992 & 36 \\
\hline & Notary & Hash & OP_RETURN & $2017 / 04 / 11$ & 21 & 798 & 38 \\
\hline & Originstamp & Hash of hashes & (Commit metadata) & $2013 / 12 / 18$ & 905 & 0 & 0 \\
\hline & Btproof & Hash & (Commit metadata) & $\mathrm{N} / \mathrm{A}$ & $\mathrm{N} / \mathrm{A}$ & $\mathrm{N} / \mathrm{A}$ & $\mathrm{N} / \mathrm{A}$ \\
\hline & BitcoinTimestamp & Hash & Value, Multi-in/out & $\mathrm{N} / \mathrm{A}$ & $\mathrm{N} / \mathrm{A}$ & $\mathrm{N} / \mathrm{A}$ & $\mathrm{N} / \mathrm{A}$ \\
\hline & Blocknotary & Merkle root & OP_RETURN & $\mathrm{N} / \mathrm{A}$ & $\mathrm{N} / \mathrm{A}$ & $\mathrm{N} / \mathrm{A}$ & $\mathrm{N} / \mathrm{A}$ \\
\hline & Tangible & Hash & OP_RETURN & $\mathrm{N} / \mathrm{A}$ & $\mathrm{N} / \mathrm{A}$ & $\mathrm{N} / \mathrm{A}$ & $\mathrm{N} / \mathrm{A}$ \\
\hline & Chainpoint & Merkle root & OP_RETURN & $\mathrm{N} / \mathrm{A}$ & $\mathrm{N} / \mathrm{A}$ & $\mathrm{N} / \mathrm{A}$ & $\mathrm{N} / \mathrm{A}$ \\
\hline & Diploma & Hash & OP_RETURN & $\mathrm{N} / \mathrm{A}$ & $\mathrm{N} / \mathrm{A}$ & $\mathrm{N} / \mathrm{A}$ & $\mathrm{N} / \mathrm{A}$ \\
\hline & Apertus & Hash & P2PKH & $\mathrm{N} / \mathrm{A}$ & $\mathrm{N} / \mathrm{A}$ & $\mathrm{N} / \mathrm{A}$ & $\mathrm{N} / \mathrm{A}$ \\
\hline & Chronobit & Hash & $\mathrm{N} / \mathrm{A}$ & $\mathrm{N} / \mathrm{A}$ & $\mathrm{N} / \mathrm{A}$ & $\mathrm{N} / \mathrm{A}$ & $\mathrm{N} / \mathrm{A}$ \\
\hline & Seclytics & Hash & OP_RETURN & $\mathrm{N} / \mathrm{A}$ & $\mathrm{N} / \mathrm{A}$ & $\mathrm{N} / \mathrm{A}$ & $\mathrm{N} / \mathrm{A}$ \\
\hline & Total & - & - & $2013 / 12 / 18$ & 198,095 & $7,502,109$ & 38 \\
\hline \multirow{5}{*}{ DRM } & Monegraph & Copyright & OP_RETURN & $2015 / 06 / 28$ & 67,286 & $2,464,282$ & 37 \\
\hline & Blockai & Copyright & OP_RETURN & $2015 / 01 / 09$ & 670 & 38,327 & 57 \\
\hline & Ascribe & Copyright & OP_RETURN & $2014 / 12 / 19$ & 48,450 & $1,000,561$ & 21 \\
\hline & Verisart & Merkle root & $\mathrm{N} / \mathrm{A}$ & $\mathrm{N} / \mathrm{A}$ & $\mathrm{N} / \mathrm{A}$ & $\mathrm{N} / \mathrm{A}$ & $\mathrm{N} / \mathrm{A}$ \\
\hline & Total & - & - & $2014 / 12 / 19$ & 116,406 & $3,503,170$ & 30 \\
\hline \multirow{7}{*}{ Message } & Eternity Wall & Text & OP_RETURN & $2015 / 06 / 24$ & 4,129 & 177,916 & 43 \\
\hline & Cryptograffiti & Text & P2PKH, Multi-in/out & $\mathrm{N} / \mathrm{A}$ & $\mathrm{N} / \mathrm{A}$ & $\mathrm{N} / \mathrm{A}$ & $\mathrm{N} / \mathrm{A}$ \\
\hline & BIT-COMM & Text & P2PKH, Multi-in/out & $\mathrm{N} / \mathrm{A}$ & $\mathrm{N} / \mathrm{A}$ & $\mathrm{N} / \mathrm{A}$ & $\mathrm{N} / \mathrm{A}$ \\
\hline & Stone & Text, File & P2PKH, Multi-in/out & $\mathrm{N} / \mathrm{A}$ & $\mathrm{N} / \mathrm{A}$ & $\mathrm{N} / \mathrm{A}$ & $\mathrm{N} / \mathrm{A}$ \\
\hline & Key.run & Magnet link & OP_RETURN & $\mathrm{N} / \mathrm{A}$ & $\mathrm{N} / \mathrm{A}$ & $\mathrm{N} / \mathrm{A}$ & $\mathrm{N} / \mathrm{A}$ \\
\hline & BitAlias & Secret, Hash & OP_RETURN & - & 0 & 0 & 0 \\
\hline & Total & - & - & $2015 / 06 / 24$ & 4,129 & 177,916 & 43 \\
\hline \multirow{5}{*}{ Subchain } & Keybase & Merkle root & OP_RETURN & $\mathrm{N} / \mathrm{A}$ & $\mathrm{N} / \mathrm{A}$ & $\mathrm{N} / \mathrm{A}$ & $\mathrm{N} / \mathrm{A}$ \\
\hline & Uniquebits & PGP signed hash & P2PKH, P2SH & $\mathrm{N} / \mathrm{A}$ & $\mathrm{N} / \mathrm{A}$ & $\mathrm{N} / \mathrm{A}$ & $\mathrm{N} / \mathrm{A}$ \\
\hline & Blockstore & Key-Value & OP_RETURN & $2014 / 12 / 10$ & 209,422 & $6,068,584$ & 29 \\
\hline & Catena [53] & Text & OP_RETURN, Tx chains & $\mathrm{N} / \mathrm{A}$ & $\mathrm{N} / \mathrm{A}$ & $\mathrm{N} / \mathrm{A}$ & $\mathrm{N} / \mathrm{A}$ \\
\hline & Total & - & - & $2014 / 12 / 10$ & 209,422 & $6,068,584$ & 29 \\
\hline Empty & Total & - & OP_RETURN & $2014 / 03 / 20$ & 296,396 & $\mathbf{0}$ & $\mathbf{0}$ \\
\hline TOTAL & - & - & - & $2009 / 01 / 03$ & $2,254,519$ & $55,066,871$ & 24 \\
\hline
\end{tabular}

Table 5: Statistics on Bitcoin-based protocols (sizes are in bytes).

\section{Discussion}

In this section we discuss the impact of metadata on the Bitcoin blockchain. We start by describing the historical evolution of metadata, highlighting how the adoption of the embedding techniques has varied over the years. We then evaluate the memory and storage consumption due to metadata, and we discuss the phenomenon of transaction peaks.

\subsection{Historical perspective}

The first piece of metadata was embedded in the genesis block by Satoshi Nakamoto, through the Coinbase technique; then, since October 2011, this technique has been used regularly by miners. In the first 3 years of Bitcoin, the most used technique for embedding data was P2PKH. Later, many protocols (e.g., Counterparty) migrated from the P2PKH to the OP_RETURN technique, and we rarely find protocols still using P2PKH. The $\mathrm{P} 2 \mathrm{PKH}$ is now used for embedding large files with the 


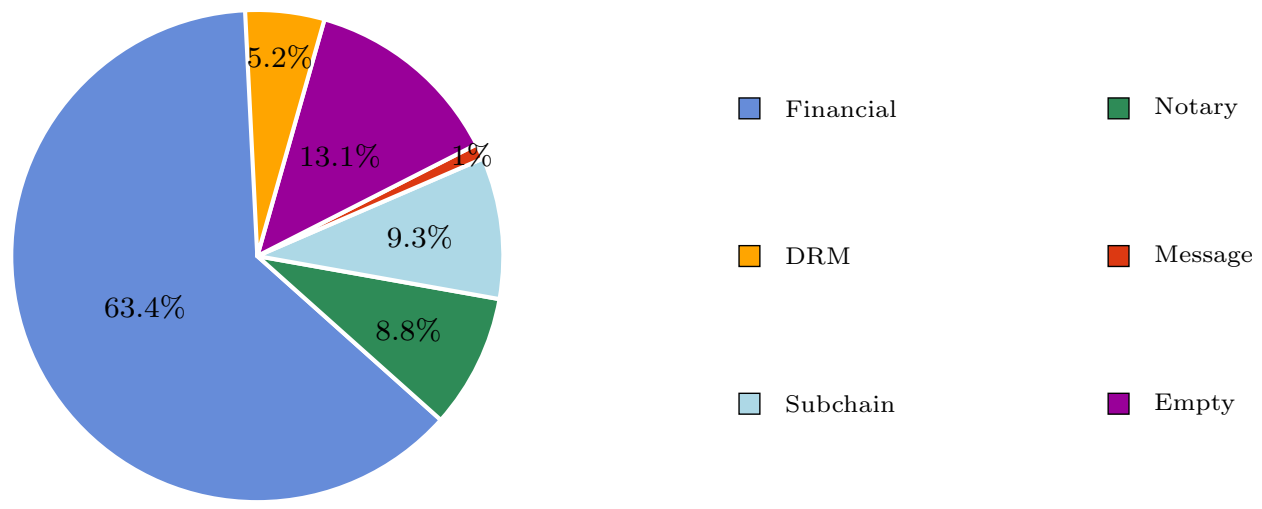

Fig. 2: Metadata transactions by protocol type.

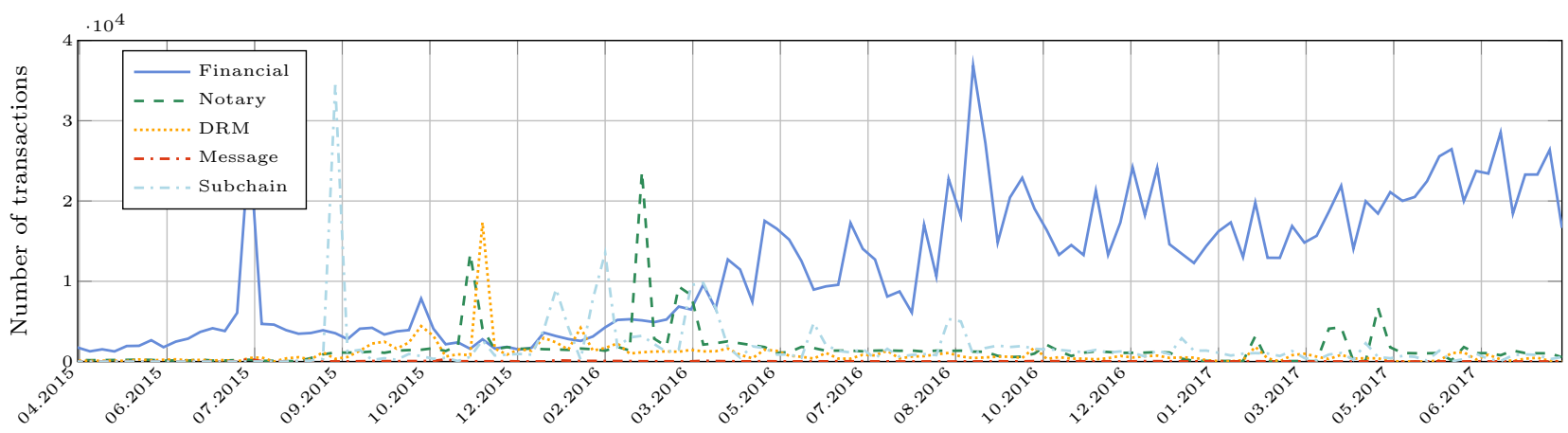

Fig. 3: Temporal evolution of metadata (transactions before April 2015 are negligible).

support of the Multi-in/out, Multi-signature, and Tx chains techniques. Despite the similarity with P2PKH, the P2PK technique is less used, since P2PK scripts are considered obsolete [10]. The Input Sequence and the Value techniques are not widely adopted as well, probably because of the limited space they offer respect to other techniques. Also the $\mathrm{P} 2 \mathrm{SH}$ technique is not widely used, although there are some proposals for adopting it for Counterparty ${ }^{9}$.

Although OP_RETURN has been part of the scripting language since the first releases of Bitcoin, originally it was considered non-standard, so transactions containing this opcode were not reliably mined. OP_RETURN became standard with Bitcoin Core 0.9.0 [8], but still the release notes state that: "This change is not an endorsement of storing data in the blockchain. The OP_RETURN change creates a provably-prunable output, to avoid data storage schemes [...] that were storing arbitrary data such as images as forever-unspendable TX outputs, bloating bitcoin's UTXO database". The limit for storing data with OP_RETURN was originally planned to be 80

\footnotetext{
9 See counterpartytalk.org/t/cip-proposal-p2sh-dataencoding/2169.
}

bytes, but the first official client supporting the opcode, i.e. the release 0.9 .0 , allowed only 40 bytes. This animated a long debate $[4,5,13,15]$. From the release 0.10 .0 [6] nodes could choose whether to accept or not OP_RETURN transactions, and set a maximum for their size. The maximum size was then set to 80 bytes by the release 0.12.0 [7]. From Table 5 we see that the majority of the applications built on top of Bitcoin embed metadata through the OP_RETURN technique; this is coherent with the data in Table 2, from which we see that $\sim 63 \%$ of the metadata in the blockchain have been embedded with the OP_RETURN technique (which is the most adopted one since March 2014). In the last period of our experiments, $\sim 40,000$ new OP_RETURN transactions are published each week. Overall, OP_RETURN transactions amount to $\sim 1,18 \%$ of the total number of transactions $(\sim 1,37 \%$ when considering the portion of the blockchain from 2014/03/12, when the first OP_RETURN transaction appeared $)^{10}$.

\footnotetext{
10 Despite the 5 years of delay, this percentage is quite close to that for the whole blockchain: this is because the number of daily transactions has largely increased since July 2014.
} 


\subsection{UTXO bloating}

As remarked in Section 3, the embedding techniques $\mathrm{P} 2 \mathrm{PK}, \mathrm{P} 2 \mathrm{PKH}$, and $\mathrm{P} 2 \mathrm{SH}$ (which are often used to embed media files), produce unspendable outputs. In this way they contribute to the "UTXO bloating" effect, that deteriorates the performance of Bitcoin nodes. In the UTXO set we have counted $\sim 68 \mathrm{~K}$ unspendable outputs which are used to embed chunks of metadata. Of all the transaction which embed metadata, only $\sim 1.49 \%$ contribute to the UTXO bloating effect.

The other embedding techniques, among which the OP_RETURN, do not bloat the UTXO (even though they still affect the total size of the blockchain). This, together with the possibility of embedding up to 80 bytes of metadata, are perhaps the reasons of the popularity of the OP_RETURN technique. Indeed, we see from Table 5 that OP_RETURN is used by the large majority of Bitcoin-based protocols. Note that the other techniques which avoid the UTXO bloating effect are not suitable to be used by protocols, either because they have a low bandwidth (Coinbase), or because they do not allow to embed enough bytes (from Table 5 we see that, on average, protocols require to embed 24 bytes of metadata).

\subsection{Space consumption}

A debated topic in the Bitcoin community is whether it is acceptable or not to save arbitrary data in the blockchain. From Table 5 we can see that the net size of metadata is $\sim 99 \mathrm{MB}$. In same period of observation, the size of the whole blockchain is $\sim 125 \mathrm{~GB}$, so the size of metadata amounts to $\sim 0.077 \%$ of the total size of transactions.

For the most widespread embedding method, the OP_RETURN, Figure 4a shows the average length of the metadata of each week. Generally, the average length of metadata is less than 40 bytes, despite the extension to 80 bytes introduced on $2015 / 07 / 12$. Peaks down on the same period are related to the Empty transactions, discussed later on in Section 6.4. Figure 4b represents the number of OP_RETURN transactions with a given data length: also this chart confirms a small number of transactions that use more than the half of the available space. Note that the discussed peak appears also in this chart, in correspondence of the 0 value. From the last column of Table 5 we see that even the protocol which embeds the largest number of bytes (Blockai, with 57 bytes on average), requires much less than the 80 bytes available with OP_RETURN. Several Notary protocols take 40 bytes on average: 16 bytes for their identifiers, and the remaining bytes for the hash they save. Gener- ally, Notary protocols carry longer metadata than the other protocols.

We now estimate the overall size of OP_RETURN transactions (including both the metadata and the other parts of the transaction). The size of an Empty transaction with one input and one output is 156 bytes. From Table 2 we see that OP_RETURN transaction carry 26 bytes of metadata, on average. We then approximate the average size of an OP_RETURN transaction as 182 bytes. Multiplying by the number of OP_RETURN transaction, we obtain an approximation of their space consumption as $\sim 503 \mathrm{MB}$.

\subsection{Transaction peaks}

Figure 5 represents peaks of OP_RETURN transactions from 2014/03 (date of the first OP_RETURN transaction) to $2017 / 08$. For each week, it shows (i) the number of Empty transactions, (ii) the number of OP_RETURN transactions which are not produced to any protocol (among those in our collection), and (iii) the total number of OP_RETURN transactions. In the graph we note several peaks, that we explain as follows:

1. $\sim 100 \mathrm{~K}$ transactions from $2015 / 07 / 08$ to $2015 / 08 / 05$. This peak is mainly composed of two different peaks of Empty transactions: the July peak $(\sim 37 \mathrm{~K}$ transactions from $2015 / 07 / 08$ to $2015 / 07 / 10$ ) and the August peak ( $\sim 29 \mathrm{~K}$ transactions from 2015/08/01 to $2015 / 08 / 03)$. Both peaks occurred coincidentally with stress tests and spam campaigns [32] ${ }^{11}$.

2. $\sim 300 \mathrm{~K}$ transactions from $2015 / 09 / 09$ to $2015 / 09 / 23$. This second peak is the highest and longest-lasting one. As before, it is mainly caused by Empty transactions $(\sim 223 \mathrm{~K})$, although here we also observe a component of Unclassified and Blockstore transactions $(\sim 35 \mathrm{~K}$ each $)$. The work [32] detects a spike also in this period, precisely around 2015/09/13, where an anonymous group performed a stress-test on the network with a money drop. This involves a public release of private keys, with the aim to cause a big race and a consequent large number of doublespend transactions. More specifically, people used the private keys to transfer to themselves the bitcoins redeemable with these keys; since many people tried to perform these transfers simultaneously, the network was flooded with many transactions trying to double-spend the same outputs. The confirmed

11 We conjecture that Empty transactions are caused by these events. To verify this conjecture we would need to compare the transaction identifiers of our Empty transactions with the identifiers of [32], which are not publicly available. 


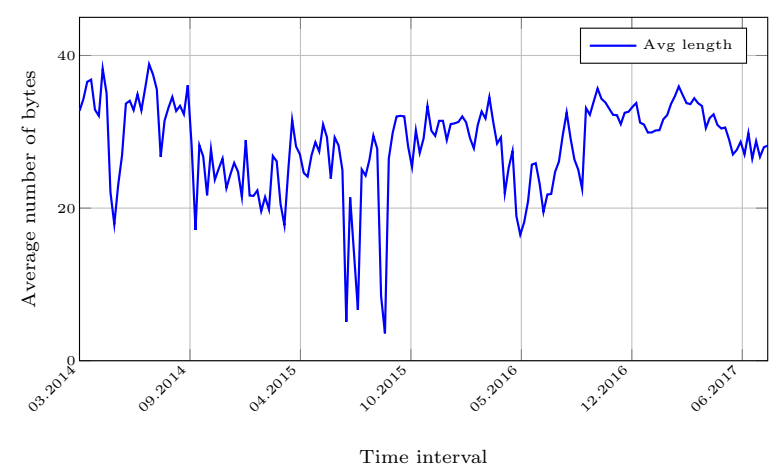

(a) Size of metadata over time.

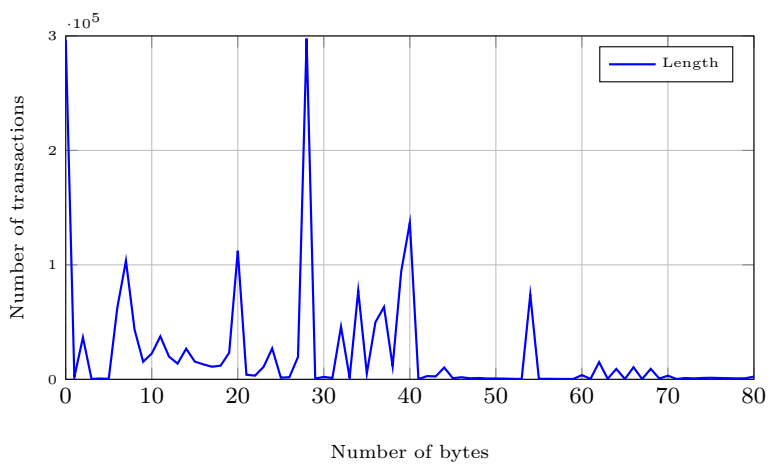

(b) Number of transactions by size of metadata.

Fig. 4: Usage and size of OP_RETURN transactions.

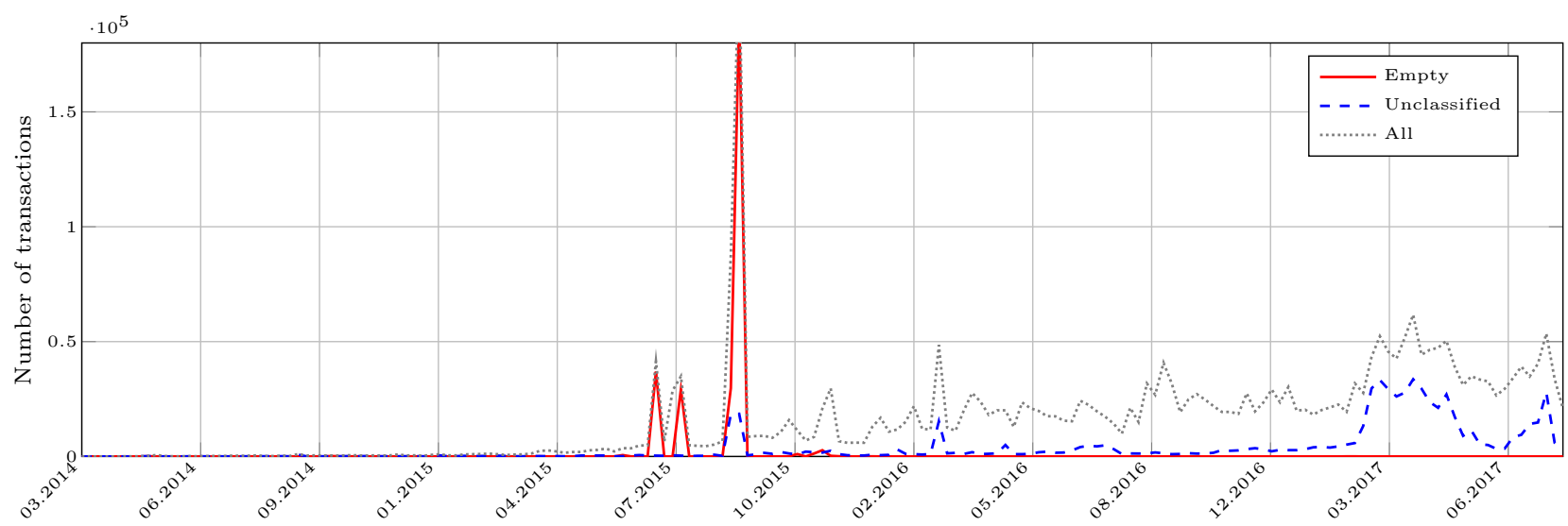

Fig. 5: Transactions (and transactions peaks) over time.

transactions caused a peak, which happened simultaneously to the peak of OP_RETURN we measured.

3. $\sim 50 \mathrm{~K}$ transactions from $2016 / 03 / 02$ to $2016 / 03 / 09$. This last peak is given by the sum of two different peaks: Unclassified $(\sim 18 \mathrm{~K})$ and Stampery $(\sim 23 \mathrm{~K})$ transactions. The part of the peak caused by Stampery can be explained as follows. Being a notarization protocol, Stampery receives document hashes off-chain, and subsequently it embeds these hashes in transactions. Since Stampery has only a few transactions before 2016/03/02 (probably, used for testing), we conjecture that the peak coincides with its bootstrap, when the protocol publishes on the blockchain all the transactions related to the hashes accumulated off-chain. The other part of the peak could be due to the bootstrap of other protocols.

Besides the peaks of OP_RETURN transactions, we can also observe other peaks: for instance, for a duration of 100 blocks starting from 2015/05/22, Bitcoin was targeted by a stress test [2], during which the network was flooded with a large number of transactions. However, the usage of OP_RETURN transactions in this period does not seem to deviate from their normal usage.

\section{Related works}

There is a growing literature on the analysis of the Bitcoin blockchain $[32,45,47,49,50]$, and also some online services which perform statistics on Bitcoin metadata $[3,17,20,24]$. Below, we group the related works into three categories.

The first category includes online services related to Bitcoin metadata. The website opreturn.org shows some statistics about OP_RETURN transactions, organised by protocol, and statistics about their usage in a certain time frame. The website smartbit.com recognises some OP_RETURN protocols and shows statistics on them. Finally, the website kaiko.com sells data about OP_RETURN transactions.

The second category contains the works on embedding techniques. At the best of our knowledge, besides our work, this category includes only [26,46], which have been developed concurrently and independently from ours. Despite the common goals, the works $[26,46]$ differ from ours in several aspects: (i) the "Tx chains" methods and the techniques for committing metadata are described only in our work; (ii) only our work and 
[46] extract and quantify the embedded metadata; (iii) the P2SH techniques are detailed in [26]. Further differences between our work and [46] are discussed below.

The third category includes the works which analyse the types of metadata, as those in Section 4. Also in this case, the work [46] is the closest to ours: the main difference between the two works is that, while [46] is focussed on discussing the benefits and risks related to metadata (e.g. privacy violations, illegal contents), we develop a protocol-wise analysis, measuring how much (and when) metadata is embedded by each protocol, and studying which use cases they support. Further, we recognize a few types of metadata (hash, financial records, and copyright) which are not dealt with by [46].

\section{Conclusions}

Although Bitcoin does not explicitly support for embedding metadata into transactions, over the years users have devised various techniques to reach this goal. After illustrating and comparing these techniques, we have extracted all the metadata embedded up to $2017 / 08 / 10$ (first 480,000 blocks), measuring the data stored by each technique. By processing the bytes extracted from transaction metadata, we have often managed to reconstruct the original content. Overall, we have reconstructed $\sim 69 \mathrm{MB}$ of documents, out of the $\sim 99 \mathrm{MB}$ totally embedded. We have classified these documents, finding that the majority of them are records produced by financial protocols. We have reconstructed also 120 files of various kinds (among which, 108 images), for a total size of $\sim 4 \mathrm{MB}$.

We have discovered 45 protocols which embed metadata into the blockchain for developing various applications. We have identified which types of metadata they produce, and which embedding techniques they use. Usually, each protocol produces one type of metadata (depending on the protocol type), using one embedding technique (most often, OP_RETURN). Overall, $\sim 53.7 \mathrm{MB}$ of metadata are produced by the protocols in our collection. The majority of protocols are for document notarization, but $\sim 70 \%$ of elements are produced by financial protocols.

Finally, we have discussed the impact of embedding metadata in the blockchain, considering various aspects, like e.g. the space consumption, the UTXO bloating effect, and the transaction peaks.

Although the official Bitcoin documentation discourages the use of the blockchain to store arbitrary data, the trend seems to be a growth in the number of applications that embed their metadata in Bitcoin transactions. We conjecture that the perceived sense of security and persistence of the Bitcoin blockchain is the main motivation to avoid using cheaper and more efficient storage. If this trend will be confirmed, the specific needs of these applications could affect the future evolution of the Bitcoin protocol.

Acknowledgements We thank Nicola Atzei for the insightful discussion on a preliminary version of this paper. This work is partially supported by Aut. Reg. of Sardinia projects Sardcoin and Smart collaborative engineering, and by COST Action IC1406 cHiPSET.

\section{References}

1. Bicoin scalability, https://en.bitcoin.it/wiki/ Scalability_FAQ. Last accessed 2018/01/01

2. Bitcoin network survives surprise stress test, http: //www . coindesk. com/bitcoin-networksurvives-stress-test/. Last accessed 2018/01/01

3. Bitcoin OP_RETURN wiki page, https://en.bitcoin.it/ wiki/OP_RETURN. Last accessed 2018/01/01

4. Bitcoin pull request 5075, https://github.com/ bitcoin/bitcoin/pull/5075. Last accessed 2018/01/01

5. Bitcoin pull request 5286, https://github.com/ bitcoin/bitcoin/pull/5286. Last accessed 2018/01/01

6. Bitcoin release 0.10 .0 , https://bitcoin.org/en/ release/v0.10.0. Last accessed 2018/01/01

7. Bitcoin release 0.12 .0 , https://bitcoin.org/en/ release/v0.12.0. Last accessed 2018/01/01

8. Bitcoin release 0.9.0, https://bitcoin.org/en/release/ v0.9.0. Last accessed 2018/01/01

9. Bitcoin script interpreter, https: //github.com/bitcoin/bitcoin/blob/ fcf646c9b08e7f846d6c99314f937ace50809d7a/src/ script/interpreter.cpp. Last accessed 2018/01/01

10. Bitcoin wiki script, https://en.bitcoin.it/wiki/ Script. Last accessed 2018/01/01

11. Bitcoin wiki transaction, https://en.bitcoin.it/wiki/ Transaction. Last accessed 2018/01/01

12. Colu website, https://www.colu.com/. Last accessed 2018/01/01

13. Counterparty open letter and plea to the Bitcoin core development team, http://counterparty.io/news/anopen-letter-and-plea-to-the-bitcoin-coredevelopment-team/. Last accessed 2018/01/01

14. Counterparty website, http://counterparty.io/. Last accessed 2018/01/01

15. Developers battle over bitcoin block chain, http://www . coindesk. com/developers-battlebitcoin-block-chain/. Last accessed 2018/01/01

16. Factom website, https://www.factom.com/. Last accessed 2018/01/01

17. Kaiko data store, https://www.kaiko.com/. Last accessed 2018/01/01

18. Omni website, http://www.omnilayer.org/. Last accessed 2018/01/01

19. Open assets website, https://github.com/OpenAssets/. Last accessed 2018/01/01

20. opreturn.org, http://opreturn.org/. Last accessed 2018/01/01

21. Proof of existence website, https://proofofexistence. com/. Last accessed 2018/01/01 
22. Scalability debate ever end, https://www. cryptocoinsnews.com/will-bitcoin-scalabilitydebate-ever-end/. Last accessed 2018/01/01

23. Scaling debate in Reddit, http://www.coindesk.com/ viabtc-ceo-sparks-bitcoin-scaling-debate-redditama/. Last accessed 2018/01/01

24. Smartbit OP_RETURN statistics, https ://www.smartbit. com.au/op-returns. Last accessed 2018/01/01

25. Stampery blockchain timestamping architecture, https: //s3.amazonaws. com/stampery-cdn/docs/StamperyBTA-v6-whitepaper.pdf. Last accessed 2018/01/01

26. Data insertion in Bitcoin's blockchain (2017), http://digitalcommons.augustana.edu/cgi/ viewcontent. cgi? article=1000\&context=cscfaculty. Last accessed 2018/01/01

27. Andresen, G.: Block v2, height in coinbase, BIP 034, https://github.com/bitcoin/bips/blob/master/ bip-0034.mediawiki. Last accessed 2018/01/01

28. Antonopoulos, A.M.: Mastering Bitcoin: unlocking digital cryptocurrencies. O'Reilly Media, Inc. (2014)

29. Atzei, N., Bartoletti, M., Cimoli, T., Lande, S., Zunino, R.: SoK: unraveling Bitcoin smart contracts. In: Principles of Security and Trust (POST). LNCS, vol. 10804, pp. 217-242. Springer (2018)

30. Atzei, N., Bartoletti, M., Lande, S., Zunino, R.: A formal model of Bitcoin transactions. In: Financial Cryptography and Data Security (2018)

31. Badertscher, C., Maurer, U., Tschudi, D., Zikas, V.: Bitcoin as a transaction ledger: A composable treatment. In: CRYPTO. LNCS, vol. 10401, pp. 324-356. Springer (2017)

32. Baqer, K., Huang, D.Y., McCoy, D., Weaver, N.: Stressing out: Bitcoin "stress testing". In: Financial Cryptography Workshops. LNCS, vol. 9604, pp. 3-18. Springer (2016)

33. Bartoletti, M., Bracciali, A., Lande, S., Pompianu, L.: A general framework for blockchain analytics. In: Proc. 1st Workshop on Scalable and Resilient Infrastructures for Distributed Ledgers (SERIAL@Middleware). pp. 7:1-7:6. ACM (2017), https://github.com/bitbart/blockapi

34. Bartoletti, M., Pompianu, L.: An analysis of Bitcoin OP_RETURN metadata. In: Financial Cryptography Workshops. LNCS, vol. 10323, pp. 218-230. Springer (2017)

35. Bartoletti, M., Pompianu, L., Bellomy, B.: Bitcoin metadata (2018), https://doi.org/10.7910/DVN/MOLW81

36. Bartoletti, M., Zunino, R.: BitML: a calculus for Bitcoin smart contracts. In: ACM CCS (2018)

37. Bellomy, B.: Binary grep, htps://github.com/ spooktheducks/binary-grep

38. Bellomy, B.: Local blockchain parser, https://github. com/spooktheducks/local-blockchain-parser

39. Bistarelli, S., Mercanti, I., Santini, F.: An analysis of nonstandard Bitcoin transactions. In: Crypto Valley Conference on Blockchain Technology (2018)

40. Bonneau, J., Miller, A., Clark, J., Narayanan, A., Kroll, J.A., Felten, E.W.: SoK: Research perspectives and challenges for Bitcoin and cryptocurrencies. In: IEEE Symp. on Security and Privacy. pp. 104-121 (2015)

41. devttys0: binwalk, https://github.com/devttys0/ binwalk

42. Garay, J.A., Kiayias, A., Leonardos, N.: The Bitcoin backbone protocol: Analysis and applications. In: EUROCRYPT. LNCS, vol. 9057, pp. 281-310. Springer (2015)

43. Gerhardt, I., Hanke, T.: Homomorphic payment addresses and the pay-to-contract protocol. arXiv preprint arXiv:1212.3257 (2012)
44. Kosba, A.E., Miller, A., Shi, E., Wen, Z., Papamanthou, C.: Hawk: The blockchain model of cryptography and privacy-preserving smart contracts. In: IEEE Symp. on Security and Privacy. pp. 839-858 (2016)

45. Lischke, M., Fabian, B.: Analyzing the Bitcoin network: The first four years. Future Internet 8(1), 7 (2016)

46. Matzutt, R., Hiller, J., Henze, M., Ziegeldorf, J.H., Mullmann, D., Hohlfeld, O., Wehrle, K.: A quantitative analysis of the impact of arbitrary blockchain content on Bitcoin. In: Financial Cryptography and Data Security (2018)

47. Möser, M., Böhme, R.: Trends, tips, tolls: A longitudinal study of Bitcoin transaction fees. In: Financial Cryptography and Data Security. LNCS, vol. 8976, pp. 19-33. Springer (2015)

48. Nakamoto, S.: Bitcoin: a peer-to-peer electronic cash system. https://bitcoin.org/bitcoin.pdf (2008)

49. Reid, F., Harrigan, M.: An analysis of anonymity in the Bitcoin system. In: Security and privacy in social networks, pp. 197-223. Springer (2013)

50. Ron, D., Shamir, A.: Quantitative analysis of the full Bitcoin transaction graph. In: Financial Cryptography and Data Security. LNCS, vol. 7859, pp. 6-24. Springer (2013)

51. Stevens, M., Bursztein, E., Karpman, P., Albertini, A., Markov, Y.: The first collision for full SHA-1. In: CRYPTO. LNCS, vol. 10401, pp. 570-596. Springer (2017)

52. Todd, P.: Delayed TXO commitments, https: //petertodd.org/2016/delayed-txo-commitments. Last accessed 2018/01/01

53. Tomescu, A., Devadas, S.: Catena: Efficient nonequivocation via Bitcoin. In: IEEE Symp. on Security and Privacy. pp. 393-409 (2017) 